\title{
Research on the frequency of post-traumatic stress disorder in healthcare workers during the COVID-19 pandemic
}

\author{
Süleyman Korkut ${ }^{1}$ D
}

Received: 12 June 2021 / Accepted: 24 November 2021 / Published online: 1 December 2021

(c) The Author(s), under exclusive licence to Royal Academy of Medicine in Ireland 2021

\begin{abstract}
Background It is highly probable that the COVID-19 outbreak, one of the most severe pandemics to which humanity has been exposed, will promote post-traumatic stress disorder (PTSD). PTSD is a serious mental illness that decreases quality of life and functionality of healthcare workers (HCWs) during the COVID-19 pandemic.

Aims This study aims to research the frequency of PTSD in HCWs who are working in a central pandemic hospital, during the COVID-19 outbreak.

Methods In total, $300 \mathrm{HCWs}$ were included in the study. Diagnosis of PTSD was made according to DSM-5 diagnostic criteria, and the severity of PTSD symptoms was evaluated using the PTSD Checklist - Civilian Version Scale.

Results In total, $21.6 \%(n=65)$ of the HCWs were diagnosed with PTSD, 18.3\% of them were female $(n=55)$ and $3.3 \%$ were male $(n=10)$ participants. The mean PCL-C score of participants diagnosed with PTSD was found to be $60.38 \pm 4.81$. No statistically significant difference was found between profession groups diagnosed with PTSD.

Conclusions HCWs who are working directly with COVID-19 patients have significantly higher levels of PTSD. PTSD can lead to severe negative consequences such as lower quality of life and loss of workforce and productivity, if it is not diagnosed and treated early. Therefore, it should become a routine to continuously monitor and establish early targeted mental health interventions.
\end{abstract}

Keywords COVID-19 $\cdot$ Healthcare workers $\cdot$ Post-traumatic stress disorder

\section{Introduction}

COVID-19 (coronavirus disease-19) was first detected in a province in China in December 2019 and then spread rapidly in many regions of China and from there to almost all countries in the world. COVID-19 was declared a pandemic (global epidemic) by the World Health Organization on March 11, 2020 [1]. Mental symptoms due to increasing workload and responsibility of HCWs (healthcare workers) are excessively increasing in the COVID-19 pandemic similar to previous pandemics. While HCWs are heroically fighting against the pandemic, they also have to fight against the psychological effects of an outbreak. Since the first cases of COVID-19 (coronavirus-19 disease) have emerged in China on December 8, 2019, the disease has rapidly spread

Süleyman Korkut

dr.korkut@hotmail.com

1 University of Health Sciences, Antalya Training and Research Hospital, Antalya, Turkey to the entire world with more than one hundred thousand cases and thousands of deaths [2]. Post-traumatic stress disorder (PTSD) is a common mental disorder among people who experienced a serious trauma such as war, violence, natural disaster, traffic or occupational accident, and fatal communicable disease epidemic. It is a mental disorder characterized with constantly reliving the stated situation, negative changes in cognition and mood, constant avoidance from stimulus accompanying the trauma, and accompanying signs of increased arousal which starts following a traumatic (destructive) incident and continues for at least 1 month. The symptom of the disease starts after the traumatic incident and continues for more than 1 month [3]. Many HCWs experience severe emotional stress during the pandemic. A relevant study reported that the thought of carrying the virus and possibility of worrying about infecting other people were higher among HCWs during the SARS pandemic [4]. Another risk for many HCWs is the behavior of avoidance. It has been seen that HCWs displayed avoidance behaviors such as minimizing direct contact with patients 
and not going to work regularly [5]. Extraordinary epidemics caused PTSD in the past $[6,7]$. Considering that humanity is facing the most severe pandemic since the Spanish Flu, it is possible that the current COVID-19 pandemic will also cause PTSD [8]. This study aimed to assess the prevalence and predictive factors of post-traumatic stress among HCWs who worked in a pandemic hospital during the COVID-19 outbreak.

\section{Methods}

\section{Study population}

The study was conducted between 15 July 2020 and 15 October 2020 with a cross-sectional design. The participants were $300 \mathrm{HCWs}$ who were working at a hospital, which was determined to be a pandemic hospital in Turkey. From the formula of "the number of samples in groups with a certain number in the universe," the prevalence of PTSD was considered $20 \%$ during epidemic periods in previous population-based studies, and the lower limit number of samples to be selected from the population was calculated as 271 , with a $95 \%$ confidence interval and a margin of error of 0.05 . HCWs were evaluated into six major occupational groups and 50 people were randomly selected from each occupation equally. A sample of $300 \mathrm{HCWs}$ was determined from the population of 2037 numbered by the cluster sampling method. Interviews were made with those who agreed to participate in this study, and sociodemographic data form and SCID-5 were filled. After psychiatric interviews were made with all participants, the evaluation scales were implemented. The diagnosis of PTSD was made based on the diagnosis criteria of the Diagnostic and Statistical Manual of Mental Disorders, Fifth Edition (DSM-5) [3], and those with severe PTSD symptoms were evaluated with the "Post-Traumatic Stress Disorder Checklist - Civilian Version" (PCL-C). Those with comorbid psychiatric diagnoses and those who used and were using psychotropic medication within the last 1 month were not included in the study. The participants were informed about the study in detail and their informed consent forms were obtained before conducting the study. Ethics committee approval was obtained for the study and it was conducted in accordance with the principles stated in the Declaration of Helsinki.

\section{Data collection tools}

\section{Personal information form}

The form prepared by the researchers included age, sex, marital status, profession, and other sociodemographic characteristics and clinical characteristics.
The Structured Clinical Interview for DSM-5 Clinical Version (SCID-5-CV)

The scope of SCID-5 has expanded with changes in diagnostic categories, and its function to prevent diagnosis uncertainty and missed diagnosis has been further strengthened compared to its previous version. It has been supported that this new form is easy to apply and is useful for clinical assessments, studies, and training. It has been shown that SCID-5 can safely be used in Turkey [9].

\section{The Post-Traumatic Stress Disorder Checklist - Civilian Version (PCL-C)}

PCL-C was firstly developed based on the DSM-III PTSD diagnosis criteria, and it was reviewed after the DSM-IV and found to be valid [10]. It is a self-reporting scale with 17 items in a Likert-type structure. The first five questions show the signs of reliving, the following seven questions show the signs of avoidance, and the next five questions show the signs of increased arousal. The answers change between "none" [(0)] and "extremely" [(5)]. The PCL-C test has been successfully used to measure the PTSD symptoms of civil trauma patients [11]. It was found in relevant studies that the optimal screening cutoff score is between 31 and 50 [12-14]. In another study, test results based on the PCL-C cutoff score of $30,38,44,50$, and 60 were evaluated, and it was found that sensitivity decreased as the cutoff score increased and specificity increased as the cutoff score increased in terms of the PTSD diagnosis [10]. The Turkish validity and reliability studies of the scale were conducted and it was shown that it is a tool that can be used both in PTSD screening and evaluation of the severity of PTSD [15].

\section{Statistical analysis}

The statistical analysis of the data obtained from the study was conducted with SPSS 21.0 program. Descriptive statistics were presented as mean \pm standard deviation. The differences between means were regarded as statistically significant when the $p$ value was lower than 0.05 .

The difference between mean ages in terms of gender was analyzed with the independent samples $T$-test. The difference between those diagnosed with PTSD in terms of gender, education status, marital status, profession, living at home, having a history of psychiatric disease, having a family history of psychiatric disease, and having a chronic disease was analyzed with the chi-square test.

PCL-C mean score difference between participants diagnosed with PTSD and not diagnosed with PTSD was analyzed by an independent samples $T$-test. The difference between mean PCL-C scores in terms of gender, education status, marital status, living at home, having a history of 
psychiatric disease, having a family history of psychiatric disease, and having a chronic disease was analyzed with the independent samples $T$-test. The difference between profession groups was analyzed with the one-way ANOVA test.

\section{Results}

The study included 130 male and 170 female HCWs aged between 21 and 61 . HCWs were divided into six groups as doctor, nurse, secretary, security guard, health officer, and cleaning-cafeteria staff. Each group included the same numbers of participants in a way that the groups are similar for the homogeneity. The mean age of female participants was $37.74 \pm 8.15$ while the mean age of male participants was $37.15 \pm 9.61$. There was no statistically significant difference between female and male participants in terms of age $(p=0.573)$. Of the participants, $21.6 \%(n=65)$ were diagnosed with PTSD, and $18.3 \%$ of them were women $(n=55)$ while $3.3 \%$ were men $(n=10)$. In the study, $32.4 \%$ of female participants were diagnosed with PTSD and $7.7 \%$ of male participants were diagnosed with PTSD and there was a statistically significant difference in terms of gender $(p=0.001)$. There was no statistically significant difference found between the participants diagnosed with PTSD in terms of education, profession, having a history of psychiatric disorder, and having a chronic disease (respectively $p=0.779, p=0.138, p=0.487, p=0.257$ ). There was a statistically significant difference in terms of marital status, living at home, and having a family history of psychiatric disorder (respectively $\mathrm{p}=0.015, p=0.001, p=0.013$ ) (Table 1).

The mean PCL-C score of the participants was 41.50 \pm 13.19 . While the mean PCL-C score of the participants without the diagnosis of PTSD was $36.27 \pm 9.47$, the mean PCL-C score of the participants diagnosed with PTSD was $60.38 \pm 4.81$. The mean PCL-C scores were found to be significantly higher in those diagnosed with PTSD $(p=0.001)$. The mean PCL-C score was $45.11 \pm 14.62$ among women and $36.76 \pm 9.16$ among men. The mean PCL-C score was found to be significantly higher in women $(p=0.001)$.

The mean PCL-C score of those diagnosed with PTSD was $61.45 \pm 4.45$ among women and $54.5 \pm 0.52$ among men. The mean PCL-C score of those diagnosed with PTSD was found to be significantly higher in women $(p=0.001)$.

There was no statistically significant difference between the mean PCL-C scores of the participants in terms of education, marital status, living at home, having a history of psychiatric disorder, and having a family history of psychiatric disorder (respectively $p=0.419, p=0.313, p=0.106$, $p=0.113, p=0.108)$. There was a statistically significant

Table 1 Comparison of sociodemographic characteristics between participants diagnosed with PTSD and without PTSD

\begin{tabular}{|c|c|c|c|c|c|}
\hline & & $\begin{array}{l}\text { Diagnosed with } \\
\text { PTSD }(n)(\%)\end{array}$ & $\begin{array}{l}\text { Not diagnosed } \\
\text { with PTSD }(n)(\%)\end{array}$ & Total $(n)(\%)$ & $p$ \\
\hline \multirow[t]{2}{*}{ Gender } & Male & $10(\% 3.3)$ & $120(\% 40)$ & $130(\% 43.3)$ & \multirow[t]{2}{*}{$p=0.001$} \\
\hline & Female & $55(\% 18.3)$ & $115(\% 38.3)$ & $170(\% 56.7)$ & \\
\hline \multirow[t]{2}{*}{ Marital status } & Married & $50(\%$ 16.7) & $145(\% 48.3)$ & $195(\%$ 65) & \multirow[t]{2}{*}{$p=0.015$} \\
\hline & Single & $15(\% 5)$ & $90(\% 30)$ & $105(\% 35)$ & \\
\hline \multirow[t]{2}{*}{ Graduated university } & Yes & $30(\% 10)$ & $115(\% 38.3)$ & $145(\% 48.3)$ & \multirow[t]{2}{*}{$p=0.779$} \\
\hline & No & $35(\%$ 11.7) & $120(\% 40)$ & $155(\% 51.7)$ & \\
\hline \multirow[t]{2}{*}{ Living at home } & Alone & $5(\% 1.7)$ & $60(\% 20)$ & $65(\% 21.7)$ & \multirow[t]{2}{*}{$p=0.001$} \\
\hline & Not alone & $60(\% 20)$ & $175(\% 58.3)$ & $235(\% 78.3)$ & \\
\hline \multirow[t]{2}{*}{ Having a history of psychiatric disorder } & Yes & $15(\% 5)$ & $45(\% 15)$ & $60(\% 20)$ & \multirow[t]{2}{*}{$p=0.487$} \\
\hline & No & $50(\% 16.7)$ & $190(\% 63.3)$ & $240(\% 80)$ & \\
\hline \multirow[t]{2}{*}{ Having a family history of psychiatric disorder } & Yes & $15(\% 5)$ & $25(\% 8.3)$ & $40(\%$ 13.3) & \multirow[t]{2}{*}{$p=0.013$} \\
\hline & No & $50(\%$ 16.7) & $210(\% 70)$ & $260(\% 86.7)$ & \\
\hline \multirow[t]{2}{*}{ Having a chronic disease } & Yes & $5(\% 1.7)$ & $70(\% 23.3)$ & $75(\% 25)$ & \multirow[t]{2}{*}{$p=0.257$} \\
\hline & No & $60(\% 20)$ & $165(\% 45)$ & $225(\% 75)$ & \\
\hline \multirow[t]{6}{*}{ Profession } & Doctor & $10(\% 3.3)$ & $40(\%$ 13.4) & $50(\% 16.7)$ & \multirow[t]{6}{*}{$p=0.138$} \\
\hline & Nurse & $12(\% 4)$ & $38(\%$ 12.7) & $50(\% 16.7)$ & \\
\hline & Secretary & $11(\% 3.7)$ & $39(\% 13)$ & $50(\% 16.7)$ & \\
\hline & Security & $11(\% 3.7)$ & $39(\%$ 13) & $50(\% 16.7)$ & \\
\hline & Health officer & $10(\% 3.3)$ & $40(\%$ 13.4) & $50(\% 16.7)$ & \\
\hline & Cleaning-cafeteria staff & $11(\% 3.7)$ & $39(\% 13)$ & $50(\% 16.7)$ & \\
\hline
\end{tabular}

PTSD post-traumatic stress disorder 
difference in terms of having a chronic disease $(p=0.003)$. Variance analysis was performed to determine whether the mean PCL-C scores were different according to profession groups and a statistically significant difference was found $(F=9.248 ; p=0.001)$. Comparison of mean PCL-C scores is shown in Table 2.

\section{Discussion}

The aim of this study was to examine the frequency of PTSD among HCWs during the COVID-19 pandemic. HCWs, who are at the forefront during pandemics, are at risk in terms of mental disorders. Worries about the infection, feeling of tiredness, feeling of burnout in the workplace, and PTSD can be seen among HCWs. The fact that the contamination speed of the COVID-19 is fast and the contamination is easy is considered as a major stressor that causes negative effects on the mental health of HCWs. It is stated that this stress is an important risk factor for PTSD among HCWs [16]. Perceived danger, uncertainty, and uncontrollability perceptions during the previous SARS pandemic were reported to increase the incidence possibility of PTSD among individuals [17]. A relevant study determined that $25 \%$ of HCWs experienced PTSD during the SARS and Ebola periods [18]. A recent study reported that the rate of PTSD cases was 21\% after the Ebola outbreak [19]. The number of studies about PTSD conducted with HCWs is relatively low. A study conducted with doctors and nurses 2 months after the emergence of the SARS outbreak in 2004 found that the prevalence of PTSD was 20\% [20]. Regarding the current COVID-19 pandemic, a study conducted in China investigated 230 members of the medical staff. The incidence of PTSD was estimated at $27.39 \%$ [21]. In this study, the rate of HCWs diagnosed with PTSD was found $21.6 \%$. As in other mental illnesses, early diagnosis increases the possibility of cure in PTSD. Relevant studies showed that PTSD symptoms last more than 6 months after an incident and have a high possibility of continuance in the long term [22]. Another study has found that more than three-quarters of those who were diagnosed with PTSD right after the disaster and who were not treated were diagnosed with PTSD once again after 1 year [23]. Mak et al. (2009) conducted a study for 30 months after the SARS outbreak and found the prevalence of PTSD among the SARS victims in Hong Kong as $25.6 \%$, and this rate was higher than the rate at the beginning of the outbreak similar to the present study and relevant studies [24]. Therefore, the earlier the PTSD is diagnosed and treated, the higher the chance of the recovery of one's mental health. The sociodemographic characteristics of individuals diagnosed with PTSD were examined and possible risk factors were tried to be determined in this study. Of the HCWs who were diagnosed with PTSD, 32.4\% are within female participants and $7.7 \%$ are within male participants, and there was a significant difference in terms of gender. So PTSD was more common in women HCWs in our study.

In the study, it was found that PTSD was higher in HCWs who are married and not living alone at home. This result shows that HCWs are concerned about their families than themselves during the pandemic. In parallel with this result found in another study, one of the risks of having PTSD for HCWs is the fear of transmitting the infection to family, relatives, and friends [25].

In the study, there was no significant difference between the profession groups among those diagnosed with PTSD. On the other hand, the mean PCL-C score of nurses was
Table 2 Comparison of mean PCL-C scores

\begin{tabular}{llll}
\hline & & $\begin{array}{l}\text { The mean PCL-C score } \\
(m \pm s d)\end{array}$ & $p$ \\
\hline Gender & Female & $45.11 \pm 14.62$ & $p=0.001^{*}$ \\
& Male & $36.76 \pm 9.16$ & \\
Total & $41.50 \pm 13.19$ & \\
Piagnosed with PTSD & Yes & $60.38 \pm 4.81$ & $p=0.001^{*}$ \\
& No & $36.27 \pm 9.47$ & \\
Profession & Health officer & $33.7 \pm 9.17$ & \\
& Secretary & $42.7 \pm 12.23$ & \\
& Security & $39.1 \pm 12.94$ & \\
& Cleaning-cafeteria staff & $44.2 \pm 14.28$ & \\
Having a chronic disease & Nurse & $49.4 \pm 16.89$ & \\
& Doctor & $40.4 \pm 13.47$ & \\
& Yes & $54.3 \pm 13.49$ & \\
& No & $42.1 \pm 9.27$ & \\
\hline
\end{tabular}

*T-test, **ANOVA; PTSD post-traumatic stress disorder, $P C L-C$ Post-Traumatic Stress Disorder Checklist - Civilian Version, $s d$ standard deviation 
significantly higher than that of other HCWs. Therefore, it was concluded that $\mathrm{HCW}$ s in this group were at higher risk of having PTSD. This result can be interpreted as traumatic stress is higher in HCWs who are in closer contact with risky people. Additionally, the mean PCL-C score was significantly higher in HCWs who have a chronic disease. This result can be explained as the traumatic stress caused by the fact that the COVID-19 is more fatal in chronic diseases.

This study has some limitations. First, because of the cross-sectional design of the study, the results show the psychological impact within the limited period of the pandemic. Another limitation is that it was conducted in a single center and the sample size was relatively small. Future studies will provide more comprehensive information about protecting the mental health during and after the COVID-19 pandemic with the elimination of these types of limitations.

This study will make a significant contribution to the literature in this important area, which a few studies have been done. It is very important that PTSD may lead to negative consequences such as lower quality of life, loss work force, and loss of productivity among HCWs because HCWs, who are fighting against the pandemic at the forefront and at high risk, break down both physically and mentally in time. For this reason, precautions should be taken by healthcare administrators to protect the mental health of HCWs. HCWs should have easy access to psychiatric support and treatment when needed. In addition, considering that the pandemic will continue for a long time, this process should be sustainable.

Acknowledgements The author thanks all the healthcare workers who participated in the study and Dr. Alper SINAN who helped in the statistical analysis of the study.

\section{Declarations}

Ethics approval All procedures performed in studies involving human participants were in accordance with the ethical standards of the institutional and/or national research committee and with the 1964 Helsinki declaration and its later amendments or comparable ethical standards.

Conflict of interest The author declares no competing interests.

\section{References}

1. World Health Organisation (2020) Coronavirus. https://www.who. int/health-topics/coronavirus. Accessed 23 Mar 2021

2. Dutheil F, Navel V, Clinchamps M (2020) The indirect benefit on respiratory health from the world's effort to reduce transmission of SARS - CoV-2. Chest 158(2):467-468

3. American Psychiatric Association (APA) (2013) Diagnostic and statistical manual of mental disorders (DSM-5), 5th edn. American Psychiatric Association, Washington
4. Reynolds DL, Garay JR, Deamond SL et al (2008) Understanding, compliance and psychological impact of the SARS quarantine experience. Epidemiol Infect 136:997-1007

5. Marjanovic Z, Greenglass ER, Coffey S (2007) The relevance of psychosocial variables and working conditions in predicting nurses' coping strategies during the SARS crisis: an online questionnaire survey. Int J Nurs Stud 44:991-998

6. Xu J, Zheng Y, Wang M et al (2011) Predictors of symptoms of posttraumatic stress in Chinese university students during the 2009 H1N1 influenza pandemic. Med Sci Monitor Int Med J Exp Clin Res 17:PH60-PH64

7. Cénat JM, Mukunzi JN, Noorishad P-G et al (2020) A systematic review of mental health programs among populations affected by the Ebola virus disease. J Psychosom Res 131:109966

8. Morens DM, Daszak P, Taubenberger JK (2020) Escaping Pandora's box - another novel coronavirus. N Engl J Med 382(14):1293-1295

9. Elbir M, Topbas OA, Bayad S et al (2019) Turkish adaptation and reliability study of Clinician Version of Structured Clinical Interview for DSM-5 disorders. Turk Psikiyatri Derg 30:51-56

10. Dobie DJ, Kivlahan DR, Maynard C et al (2002) Screening for post-traumatic stress disorder in female Veteran's Affairs patients: validation of the PTSD checklist. Gen Hosp Psychiatry 24(6):367-374

11. Blanchard EB, Jones-Alexander J, Buckley TC et al (1996) Psychometric properties of the PTSD checklist (PCL). Behav Res Ther 34(8):669-673

12. Andrykowski MA, Cordova MJ, Studts JL et al (1998) PTSD after treatment for breast cancer: prevalence of diagnosis and use of the PTSD Checklist - Civilian Version (PCL-C) as a screening instrument. J Consult Clin Psych 66(3):586-590

13. Smith MY, Redd W, DuHamel K et al (1999) Validation of the PTSD checklist-civilian version in survivors of bone marrow transplantation. J Trauma Stress 12(3):485-499

14. Manne SL, Du Hamel K, Gallelli K et al (1998) PTSD among mothers of pediatric cancer survivors: diagnosis, comorbidity, and utility of the PTSD checklist as a screening instrument. J Pediatric Psych 23(6):357-366

15. Kocabasoglu N, Corapcioglu OA, Yargic I et al (2005) Validity and reliability of Turkish "PTSD Checklist - Civilian Version" (PCL-C). N Symp 13:126-134

16. El-Hage W, Hingray $\mathrm{C}$, Lemogne $\mathrm{C}$ et al (2020) Health professionals facing the coronavirus disease 2019 (COVID-19) pandemic: what are the mental health risks? L'Encephale 46(3S):S73-S80

17. Wu P, Fang Y, Guan Z et al (2009) The psychological impact of the SARS epidemic on hospital employees in China: exposure, risk perception, and altruistic acceptance of risk. Can J Psychiat Revue Canadienne De Psychiatrie 54(5):302-311

18. Chew QH, Wei KC, Vasoo S et al (2020) Narrative synthesis of psychological and coping responses towards emerging infectious disease outbreaks in the general population: practical considerations for the COVID- 19 pandemic. Singapore Med J 61(7):350-356

19. Bah AJ, James PB, Bah N et al (2020) Prevalence of anxiety, depression and post-traumatic stress disorder among Ebola survivors in northern Sierra Leone: a cross-sectional study. BMC Public Health 20:1391

20. Chan AO, Huak CY (2004) Psychological impact of the 2003 severe acute respiratory syndrome outbreak on health care workers in a medium size regional general hospital in Singapore. Occup Med (Lond) 54:190-196

21. Huang Y, Zhao N (2020) Generalized anxiety disorder depressive symptoms and sleep quality during COVID-19 outbreak in China: a web-based cross-sectional survey. Psychiatry Res 288:112954. https://doi.org/10.1016/j.psychres.2020.112954 
22. Kessler RC, Sonnega A, Bromet E et al (1995) Posttraumatic stress disorder in the National Comorbidity Survey. Arch Gen Psychiatry 52(12):1048-1060

23. North CS, Kawasaki A, Spitznagel EL et al (2004) The course of PTSD, major depression, substance abuse and somatization after a natural disaster. J Nerv Ment Dis 192(12):823-829

24. Mak IWC, Chu CM, Pan PC et al (2009) Long-term psychiatric morbidities among SARS survivors. Gen Hosp Psychiatry 31(4):318-326
25. Houghton C, Meskell P, Delaney H et al (2020) Barriers and facilitators to healthcare workers' adherence with infection prevention and control (IPC) guidelines for respiratory infectious diseases: a rapid qualitative evidence synthesis. Cochrane Database Syst Rev 4(4)

Publisher's Note Springer Nature remains neutral with regard to jurisdictional claims in published maps and institutional affiliations. 